\title{
Existence of $k$-Edge Connected Ordinary Graphs With Prescribed Degrees
}

\author{
Jack Edmonds
}

\author{
(February 25, 1964)
}

\begin{abstract}
An ordinary graph $G$ is a set of objects called nodes and a family of unordered pairs of the nodes called edges. The degree of a node in $G$ is the number of edges in $G$ which contain it. $G$ is called connected if it is not the union of two disjoint nonempty subgraphs. A graph $H$ is called $k$-edge connected if deleting any fewer than $k$ edges from $H$ leaves a connected graph. It is proved that there exists a $k$-edge connected graph $H$ for $k>1$ with prescribed integer degrees $d_{i}$ if and only if there exists an ordinary graph with these degrees and all $d_{i} \geqslant k$. There exists a l-connected (i.e., connected) ordinary graph with prescribed positive integer degrees $d_{i}$ if and only if there exists an ordinary graph with these degrees and $\sum_{i=1}^{n} d_{i} \geqslant 2(n-1)$.
\end{abstract}

An ordinary graph $G$ is a finite set of objects and a family of two-member subsets of the objects. The objects are called the nodes of $G$ and the pairs are called the edges of $G$. An edge and a node are said to meet if one contains the other. The degree of a node in $G$ is the number of edges in $G$ which it meets.

A cut of graph $G$, denoted by $(S, \bar{S})$, is a partition of the nodes of $G$ into two nonempty subsets $S$ and $\bar{S}$. The union of $S$ and $\bar{S}$ is all the nodes of $G$. The intersection of $S$ and $S$ is empty. An edge $e$ in cut $(S, \bar{S})$ of $G$ is an edge of $G$ which meets one node in $S$ and one node in $\bar{S}$. The value of cut $(S, \bar{S})$ is the number of edges in it.

Graph $G$ is called $k$-edge connected if it has no cut with value less than $k$. The usual meaning of connected for graphs is the same as the meaning of 1 edge connected. An equivalent definition of $k$-edge connected for $G$ is that $G$ cannot be made nonconnected by deleting fewer than $k$ edges from $G$.

Hoffman, Fulkerson, and McAndrew ${ }^{2}$ describe necessary and sufficient conditions for a set of integers to be the degrees of the nodes of some ordinary graph, and give references to related work. Here, we show that simple additional conditions are necessary and sufficient for the set of integers to be the degrees of the nodes of some $k$-edge connected ordinary graph (prescribed $k$ ).

THEOREM. Integers $\mathrm{d}_{\mathrm{i}}, \mathrm{i}=1, \ldots, \mathrm{n},(\mathrm{n}>1)$, are the respective degrees of the nodes $\mathrm{v}_{\mathrm{i}}$ of some $\mathrm{k}$-edge connected ordinary graph if and only if

(1) they are the degrees of the nodes of some ordinary graph,

(2) all $\mathrm{d}_{\mathrm{i}} \geqslant \mathrm{k}$,

(3) where $\mathrm{k}=1, \sum_{i=1}^{n} d_{i} \geqslant 2(\mathrm{n}-1)$.

${ }^{1}$ Supported by the Army Research Office (Durham).

${ }^{2}$ D. R. Fulkerson, A. J. Hoffman, and M. H. McAndrew, Some properties of graphs with multiple edges, to appear in the Canadian Journal of Mathematics.
The "only if" part of the theorem is almost obvious. Certainly condition ( 1 ) is necessary. Condition (2) is necessary since a cut of a graph $G$ which puts one node, say $v$, in one part and the other nodes of $G$ in the other part has cut value equal to the degree of $v$.

For $k=1$, condition (2) - that the $d_{i}$ 's be positiveis necessary but not sufficient. Let $G$ be a connected graph with $n$ nodes. There must be an edge, say $e_{1}$, joining node $v_{1}$ of $G$ to one of the other nodes of $G$, say $v_{2}$. Recursively, there must be an edge, say $e_{i}$, joining one of the nodes $v_{1}, \ldots, v_{i}(1 \leqslant i \leqslant n-1)$ of $G$ to one of the other nodes of $G$, say $v_{i+1}$. Clearly each of the edges $e_{i}(i=1, \ldots, n-1)$ is distinct. Therefore the number of edges in $G$ is not less than $n-1$. Let $d_{i}$ be the degree of node $v_{i}(i=1, \ldots, n)$ in $G$. Because each edge of $G$ contributes twice to a degree in $G, \Sigma d_{i}$ equals twice the number of edges in $G$. Therefore, $\Sigma d_{i} \geqslant 2(n-1)$.

A (simple) path joining nodes $a$ and $b$ is a connected graph which has degree $=1$ at its nodes $a$ and $b$ and degree $=2$ at its other nodes. The edges $e_{i}(i=1, \ldots, n-1)$ described in the last paragraph form with their nodes a tree. A tree is a graph which contains exactly one path joining each pair of its nodes. A tree contains one less edge than nodes. A graph $G$ contains a tree containing all the nodes of $G$ if and only if $G$ is connected. The edges and nodes of any graph partition uniquely into one or more connected component graphs. Two nodes in a graph $G$ are joined by a path in $G$ if and only if they are in the same component of $G$.

Suppose there exists an ordinary graph $H$, not necessarily connected, with positive integer degrees $d_{i}(i=1, \ldots, n)$ at its nodes. Suppose $\Sigma d_{i} \geqslant 2(n-1)$ and hence $H$ has at least $n-1$ edges. If $H$ is not connected, i.e., has more than one component, we shall see that another ordinary graph $H^{\prime}$ can be constructed from $H$ which has the same degrees at all the nodes and which has one less component than $H$. 
Thus it will follow by induction that there exists a connected ordinary graph with these degrees.

Suppose $H$ has components $H_{1}, \ldots, H_{p}$ with $n_{1}, \ldots, n_{p}$ nodes respectively. Each $H_{j}(j=1, \ldots, p)$ contains a tree $T_{j}$ with $n_{j}-1$ edges and all $n_{j}$ nodes of $H_{j}$. Since

$$
\sum_{j=1}^{p} n_{j}=n
$$

we have

$$
\sum_{j=1}^{p}\left(n_{j}-1\right)<n-1 \text { for } p>1 .
$$

Since there are at least $n-1$ edges in $H$, there must be an edge $e$ of $H$ not in any of the trees $T_{j}$. However $e$ must have both of its nodes, say $a$ and $b$, in one of these trees, say $T_{1}$.

Since component $\mathrm{H}_{2}$ contains a node and all $d_{i}$ 's are positive, $H_{2}$ contains an edge, say $e_{2}$, which meets nodes, say $a_{2}$ and $b_{2}$, in $H_{2}$. Form a new graph $H^{\prime}$ from $H$ by deleting from $H$ edges $e$ and $e_{2}$ and then adjoining a new edge $e_{a}$ joining $a$ and $a_{2}$ and a new edge $e_{b}$ joining $b$ and $b_{2}$. Because $a_{2}$ and $b_{2}$ are in a different component of $H$ than $a$ and $b$, there are no edges in $H$ already joining $a_{2}$ to $a$ and $b_{2}$ to $b$. Hence $H^{\prime}$ is a validly constructed ordinary graph with the same degrees at its nodes as in $H$. It is easy to verify that $H_{1}$ and $H_{2}$ are hereby replaced by a single component in $H^{\prime}$. Therefore $H^{\prime}$ has one less component than $H$. This completes the proof of the theorem for the case $k=1$.

The proof for the case $k>1$ is more tricky. Here we don't have an analog of trees or a straightforward decomposition into $k$-edge connected components to help us.

For prescribed integer $k>1$, assume that all $d_{i} \geqslant k(i=1, \ldots, n)$ and assume there exists an ordinary graph whose nodes $v_{i}$ have degrees $d_{i}$. It follows that there is a connected ordinary graph $H$ whose nodes $v_{i}$ have degrees $d_{i}$, since $d_{i} \geqslant 2$ implies

$$
\sum d_{i} \geqslant 2 n>2(n-1) \text {. }
$$

Assuming that $H$ is only $h$-edge connected where $1 \leqslant h<k$, we shall show how to construct a new ordinary graph $H^{\prime}$ whose nodes $v_{i}$ have degrees $d_{i}$, and which is at least $h$-edge connected, and which has fewer cuts of value $h$ than $H$ does. From this it will follow by induction that there is a $k$-edge connected ordinary graph $G$ whose nodes $v_{i}$ have degrees $d_{i}$.

Clearly $H$ has $h$-valued cuts $(A, \bar{A})$ and $(B, \bar{B})$ such that sets $A$ and $B$ are disjoint and such that neither $A$ nor $B$ contain a proper subset $S$ for which $(S, \bar{S})$ is only an $h$-valued cut. It may or may not be true that $A=\bar{B}$ and $\bar{A}=B$. In any case, because $H$ is connected, there must be a path $P$ joining a node, $a_{1}$, in $A$ to a node, $b_{1}$, in $B$ where $P$ has no other nodes in $A$ or $B$.

Because node $a_{1}$ has degree at least $k>h$, and because there are only $h$ edges with one node in $A$ and one node not in $A$, node $a_{1}$ must meet an edge $e_{a}$ whose other node $a_{2}$ is in $A$ and meets no edge with a node not in $A$. Similarly, node $b_{1}$ must meet an edge $e_{b}$ whose other node $b_{2}$ is in $B$ and meets no edge with a node not in $B$.
Construct a new ordinary graph $H^{\prime}$ by deleting edges $e_{a}$ and $e_{b}$ and then adjoining a new edge $e_{a}^{\prime}$ joining $a_{1}$ to $b_{2}$ and a new edge $e_{b}^{\prime}$ joining $b_{1}$ to $a_{2}$. Graph $H^{\prime}$ has the same degrees at the various nodes as $H$. The value of the cuts $(A, \bar{A})$ and $(B, \bar{B})$ is increased to $h+2$.

It remains to show that replacing $H$ by $H^{\prime}$ introduces no new cuts of value $\leqslant h$. Any cut, say $(D, \bar{D})$, whose value decreases in going from $H$ to $H^{\prime}$ must have $a_{1}$ and $b_{2}$ together in one of the parts, say $D$, and $a_{2}$ and $b_{1}$ together in the other part, $\bar{D}$. Suppose cut $(D, \bar{D})$ in $H^{\prime}$ has value $h$ or less.

Consider the mutually disjoint sets of nodes $A \cap D, A \cap \bar{D}, B \cap D, B \cap \bar{D}$. Path $P$, defined with respect to $H$, is also entirely in $H^{\prime}$ and it joins node $a_{1}$ in $A \cap D$ to node $b_{1}$ in $B \cap \bar{D}$. Hence some edge $e_{0}$ of $P$ is an edge of the cut $(D, \bar{D})$ of $H^{\prime}$. Since $P$ contains no nodes of $A \cap \bar{D}$ or $B \cap D$, edge $e_{0}$ neither meets one node in $A \cap \bar{D}$ and one node in $A \cap D$ nor meets one node in $B \cap D$ and one node in $B \cap \bar{D}$.

Partition the edges of the cut $(D, \bar{D})$ in $H^{\prime}$ into three classes $E_{A}, E_{B}, E_{C}: E_{A}$ consisting of all edges connecting $A \cap D$ with $A \cap \bar{D}, E_{B}$ consisting of all edges connecting $B \cap D$ with $B \cap \bar{D}, E_{C}$ being formed by the remaining edges. As has been seen above, $E_{C}$ contains $e_{0}$; hence the cardinality of $E_{C}$ is at least one. Since the cardinalities of $E_{A}, E_{B}, E_{C}$ add up to at most $h$, either the cardinality of $E_{A}$ or the cardinality of $E_{B}$ must be bounded by ${ }^{3}[(h-1) / 2]$. By symmetry, suppose the former is true. Then we have, counting $e_{a}$, at most $[(h-1) / 2]+1$ edges in $H$ with a node in $A \cap D$ and a node in $A \cap \bar{D}$.

Since the value of cut $(A, \bar{A})$ in $H$ is $h$, either $A \cap D$ or $A \cap \bar{D}$ contains nodes of no more than $[h / 2]$ edges of cut $(A, \bar{A})$. Every edge of $H$ with precisely one node in $A \cap D$ (in $A \cap \bar{D})$ has its other node in $A \cap \bar{D}$ (in $A \cap D$ ) or in $\bar{A}$. Therefore either cut $(A \cap D, \overline{A \cap D})$ or cut $(A \cap \bar{D}, \overline{A \cap \bar{D}})$ of $H$ has value at most

$$
[h / 2]+[(h-1) / 2]+1=h .
$$

However this contradicts the fact that $A$ contains no proper subset $S$ such that cut $(S, \bar{S})$ in $H$ has value at most $h$. Therefore, our assumption that cut $(D, \bar{D})$ in $H^{\prime}$ has value at most $h$ is incorrect. Therefore replacing $H$ by $H^{\prime}$ introduces no new cuts of value at most $h$. The theorem is proved.

Mr. Alvin Owen (in conversation) has posed an interesting related problem. What are necessary and sufficient conditions for a set of integers

$$
\left\{d_{i}\right\}(i=1, \ldots, n)
$$

so that all ordinary graphs whose nodes have degrees precisely $\left\{d_{i}\right\}$ are connected? Or, looking for the negative of these conditions, what are necessary and sufficient conditions for $\left\{d_{i}\right\}$ to be the degrees of the nodes of some nonconnected ordinary graph?

Note added in proof: D. R. Fulkerson has called to my attention a related paper by himself and L. S. Shapley, Minimal $k$-arc connected graphs, RAND Report P-2371 (1961). Their main result states that the fewest edges required in a $k$-edge connected ordinary graph with $n$ nodes is $[k n-1) / 2]$.

(Paper 68B2-120)

${ }^{3}$ Here $[x]$ means "the greatest integer not greater than $x . "$ 